\title{
Forking Paths ANd Polarity Items Licensing*
}

\author{
Francesca Panzeri \\ Università degli Studi di Milano-Bicocca \\ francesca.panzeri@unimib.it
}

\begin{abstract}
There is an elegant account, proposed by Beaver and Condoravdi (2003), that assumes that the temporal connectives before and after are converses (i.e., they are analyzed by means of a unified lexical schema), and that explains away their different logical and veridical behavior appealing to other factors. There is an elegant explanation that connects the licensing of Polarity Items to informational strengthening requirements: Polarity Items are viewed as existentials that lead to a widening of the domain of quantification, and they are predicted to be legitimate only when this widening leads to a stronger statement (roughly, in downward monotone contexts). My plan is to connect these two approaches - by proposing an amendment in the definition Beaver and Condoravdi presented for before and after that is meant to account also for their Polarity Items licensing behavior.
\end{abstract}

\section{The data}

It is a well-known fact that the two temporal connectives after and before appear to be converses (i.e., if (1) is true, then also (2) is true):

Fred came home after Wilma left.

Wilma left before Fred came home.

but, on the other hand, display different properties. In particular, they exhibit different logical properties: after expresses a relation which is neither transitive nor asymmetrical; before expresses a relation which is transitive and asymmetrical. ${ }^{1}$ And they have diverging veridical properties: after constitutes a veridical operator, that is, from the truth of $A$ after $B, B$ may be inferred:

(3) Fred came home after Wilma left.

VERIDICAL

(4) Wilma left.

Whereas before may be read veridically (as in (5), where the temporal clause is implied to be true); or it may receive a non-committal interpretation (as in (6), where the subordinated clause is implied to have been likely when the main clause took place); or it may assume a counterfactual reading (as in (7), where the before-clause is implied to be false):

(5) Fred bought a Toyota before the price went up.

VERIDICAL

(6) Fred left the country before anything happened.

NON-COMMITTAL

(7) Fred died before he saw his grandchildren.

COUNTERFACTUAL

\footnotetext{
* I would like to thank Gennaro Chierchia, Carlo Cecchetto, Ivano Caponigro, Carlo Geraci and the audience at Sinn und Bedeutung $\mathrm{X}$ for insightful comments. A shorter version of this paper was published in the Proceedings of the Fifteenth Amsterdam Colloquium.

${ }^{1}$ In this paper, I will not analyze after and before logical patterns.
} 
And, finally, after and before differ also in their licensing properties. The temporal connective after does not (normally) license Polarity Items, ${ }^{2}$ and it requires indicative mood (cf. (8) and the Italian (9) for explicit mood marking), whereas before does license Polarity Items, and it requires subjunctive mood (cf. (10)-(11)):

(8) * Fred left the party after anyone else did.

(9) Gianni fuggì dopo che Mario aveva rivelato (qualche / *alcun) segreto. Gianni left after that Mario had ${ }_{I N D}$ revealed (some / any) secret.

(10) Fred left the party before anyone else did.

(11) Gianni fuggì prima che Mario rivelasse alcun segreto. Gianni left before that Mario revealed ${ }_{S U B J}$ any secret.

\section{Standard account}

The traditional account may be traced back to some remarks put forth in Anscombe (1964), and it is defended, amongst others, in Landman (1991) and Ogihara (1995). Its main feature is to posit two distinct lexical entries for the temporal connectives: in both cases, the sentences $A$ after/before $B$ are regarded as true when there is a time t verifying the main clause $A$ that follows/precedes the subordinated clause $B$ - but in the case of after the $A$-time t must follow some $B$-time t' (i.e., after involves existential quantification over times verifying the temporal clause); in the case of before, the $A$-time t must precede all $B$-times t' (i.e., before requires universal quantification over times verifying the temporal clause):

\section{Landman (1991)}

$[[A$ after $B]]=1$ iff $\exists \mathrm{t}\left[\mathrm{t} \in A \& \exists \mathrm{t}^{\prime}<\mathrm{t}\left[\mathrm{t}^{\prime} \in B\right]\right]$

$A$ after $B$ is true iff there is a time t verifying $A$ and there is a time $\mathrm{t}^{\prime}$ verifying $B$, and t follows

t'. I.e., iff there is an $A$-time t that follows a $B$-time t'.

$[[A$ before $B]]=1$ iff $\exists \mathrm{t}\left[\mathrm{t} \in A \& \forall \mathrm{t}^{\prime}\left[\left(\mathrm{t}^{\prime} \in B\right) \rightarrow \mathrm{t}^{<} \mathrm{t}^{\prime}\right]\right.$

$A$ before $B$ is true iff there is a time $\mathrm{t}$ verifying $A$ and for all times $\mathrm{t}^{\prime}$, if $\mathrm{t}^{\prime}$ verifies $B$, then $\mathrm{t}$ precedes t'. I.e., iff there is an $A$-time t that precedes all $B$-times $\mathrm{t}^{\prime}$.

Within this perspective, after and before's different licensing properties immediately follow: before-clauses involve universal quantification - and thus they constitute downward entailing environments, that are known to be Polarity Items licensors; after-clauses, on the other hand, call for existential quantification over times, and thus they do not allow strengthening inferences. As for their veridicality problems, after turns out to be a veridical operator (since the instantiation of $B$ is a necessary condition for the truth of $A$ after $B$ ); whereas before is non-veridical (since for $A$ before $B$ to be true, $B$ needs not be realized). ${ }^{3}$ But the standard account has also some shortcomings: in particular, the apparent converseness between before and after is lost, and it is not clear how to present a compositional account of their meanings.

\section{Beaver and Condoravdi (2003)}

In a recent paper, ${ }^{4}$ Beaver and Condoravdi defended a uniform account for the analysis of the two temporal connectives. The first step consists in the introduction of a coercion operator earliest - that applies to a set of times verifying a clause $C$, and that selects its left boundary (i.e., the earliest amongst all the $C$-times). Sentences of the form $A$ after (/ before) $B$ are

\footnotetext{
${ }^{2}$ Linebarger presented some counterexamples to this generalization. They are discussed in the Appendix.

${ }^{3}$ Some adjustments are needed, because otherwise any sentence with an unrealized before-clause is predicted to be true - independently of its likelihood. See Ogihara (1995).

${ }^{4}$ Beaver D. \& Condoravdi C. (2003). A Uniform Analysis of Before and After.
} 
viewed as true relatively to a time $\mathrm{t}_{0}$ just in case there is a time $\mathrm{t}$ that verifies the main clause $A$ and that follows (/ precedes) the earliest time t' that verifies the temporal clause $B$.

As it stands, the definition cannot explain after and before diverging veridical properties since for the truth of $A$ before $B$ there must be a (earliest) time verifying $B$ (that is, before turns out to be a veridical operator). Beaver and Condoravdi's solution is to exploit the definedness requirement associated with the coercion operator earliest: earliest must pick up the earliest amongst all the times verifying the $B$-clause. If there are no $B$-times in the evaluation world, alternative worlds are to be taken into consideration. These alternative worlds are the historical alternatives to an evaluation world $\mathrm{w}$ at a time $\mathrm{t}-\operatorname{alt}(w, t)-$ those worlds that coincide with w up to $t$, and from that moment may diverge only in reasonable ways, i.e., the normal future continuations of $w$ after $t$. The operator earliest is then defined relatively to this expanded domain of worlds.

\section{Beaver and Condoravdi (2003)}

$$
\begin{array}{ll}
\operatorname{alt}(w, t)=\lambda w^{\prime} . & w^{\prime} \text { is indistinguishable from } w \text { for all times } \mathrm{t}^{\prime}<\mathrm{t} ; \\
\text { and } \mathrm{w}^{\prime} \text { is a normal continuation of } \mathrm{w} \text { after } \mathrm{t} .
\end{array}
$$

$[[A \text { after (/before) } B]]^{\mathrm{w}}=1$ iff $\left(\exists \mathrm{t}:\langle\mathrm{w}, \mathrm{t}>\in A) \mathrm{t}>(/<)\right.$ earliest. $\left.\lambda \mathrm{t}^{\prime} .\left(\exists \mathrm{w}^{\prime} \in \operatorname{alt}(\mathrm{w}, \mathrm{t})\right)<\mathrm{w}^{\prime}, \mathrm{t}^{\prime}\right\rangle \in B$

$A$ after (/before) $B$ is true in $\mathrm{w}$ iff there is an $A$-time $\mathrm{t}$ that follows (/precedes) the earliest amongst the times $\mathrm{t}^{\prime}$ for which there is an historical alternative $\mathrm{w}^{\prime}$ to $(\mathrm{w}, \mathrm{t})$ such that $\left\langle\mathrm{w}^{\prime}, \mathrm{t}^{\prime}\right\rangle$ verify $B$; i.e. iff there is an $A$-time t that follows (/precedes) the earliest $B$-time - not necessarily in the evaluation world $w$, but possibly in one of its historical alternative w'.

The difference between before and after's veridical properties is couched on the asymmetry of time branching: roughly, once a time $\mathrm{t}$ is located (i.e., the time in which the main clause $A$ holds), what is past with respect to $t$ is fixed - and thus the set of historical alternatives to $\mathrm{w}$ at $t$ is in fact reduced to the evaluation world $w$ itself, whereas what is future with respect to $t$ may involve different future branches, i.e., it calls for a set of historical alternative worlds.

Somehow more formally, in the evaluation of a sentence of the form $A$ after $B$, since the historical alternatives coincide with $\mathrm{w}$ for all $\mathrm{t}^{\prime}<\mathrm{t}$ (all times $\mathrm{t}^{\prime}$ that precede $\mathrm{t}$ ), and since the earliest $B$-time is located before the $A$-time t, the set $\operatorname{alt}(\mathrm{w}, \mathrm{t})$ is reduced to the singleton $\{\mathrm{w}\}$, and the definition can be simplified to:

$$
\left.[[\mathrm{A} \text { after } \mathrm{B}]]^{\mathrm{w}}=1 \text { iff }(\exists \mathrm{t}:\langle\mathrm{w}, \mathrm{t}\rangle \in A) \mathrm{t}\right\rangle \text { earliest. } \lambda \mathrm{t}^{\prime} .\left\langle\mathrm{w}, \mathrm{t}^{\prime}\right\rangle \in B
$$

For the sentence to be true, there must be an $A$-time t that follows the earliest amongst the times $\mathrm{t}^{\prime}$ that verify $B$ in the evaluation world $w$. Thus, for the sentence to be true, the subordinated clause $B$ has to be instantiated in the evaluation world - that is, after is predicted to be veridical.

When we turn to before-sentences, the situation is different. Since the event in the $B$-clause is future with respect to the $A$-time t, historical alternatives (i.e., future branches) of w after $\mathrm{t}$ are activated: $B$ is to be instantiated in at least one of these branches - not necessarily in the evaluation world.

$$
[[A \text { before } B]]^{\mathrm{w}}=1 \text { iff }(\exists \mathrm{t}:\langle\mathrm{w}, \mathrm{t}\rangle \in A) \mathrm{t}\left\langle\text { earliest. } \lambda \mathrm{t}^{\prime} .\left(\exists \mathrm{w}^{\prime} \in \operatorname{alt}(\mathrm{w}, \mathrm{t})\right)\left\langle\mathrm{w}^{\prime}, \mathrm{t}^{\prime}\right\rangle \in B\right.
$$

$A$ before $B$ is true in a world $\mathrm{w}$ if and only if there is a time $\mathrm{t}$ such that the pair $\langle\mathrm{w}, \mathrm{t}\rangle$ verifies $A$, and that time $\mathrm{t}$ precedes the earliest amongst the times $\mathrm{t}^{\prime}$ for which there is a historical alternative w' to $w$ at $t$ such that $\left\langle\mathrm{w}^{\prime}, \mathrm{t}^{\prime}\right\rangle$ verifies $B$.

Thus, for instance, coming back to the counterfactual reading of before, the sentence in (7) is predicted to be true just in case there is a past time $t$ in which Fred dies, and in at least one 
future alternative to $\mathrm{w}$ at $\mathrm{t}$ Fred sees his grandchildren, ${ }^{5}$ and that time $\mathrm{t}$ precedes the earliest amongst all the times in which he sees his grandchildren.

I think that Beaver and Condoravdi's proposal is extremely convincing, since it can explain the apparently diverging properties after and before display by means of a single lexical schema. The problem is that, in their (2003) SALT paper, after and before's different licensing behaviour remains unaccounted for. ${ }^{6}$

\section{The proposal}

The evaluation of a before-clause may require considering alternative worlds; an after-clause is assessed with respect to the evaluation world. I propose to connect the licensing of Polarity Items precisely to this difference.

This is formally obtained by introducing an amendment to Beaver and Condoravdi's uniform definition for after and before: roughly, the time t that verifies the main clause $A$ is to be ordered (as temporally following or preceding) all the earliest B-times (i.e., all the times $\mathrm{t}^{\prime}$ that constitute the earliest times verifying the $B$-clause relatively to the historical continuations of $<\mathrm{w}, \mathrm{t}\rangle)$. In other words, the new "basic" definition for the temporal connectives after and before renders both subordinated clauses downward entailing contexts (because of the universal quantification over (earliest-)times), that is, Polarity Item licensing environments. In order to account for their diverging licensing properties, the plot is then to exploit once more the asymmetry of time branching: in the evaluation of an $A$ after $B$ sentence, since what is past with respect to a given time is fixed, the universal quantification over earliest $B$-times is in fact reduced to an ordinary existential quantification - and thus the ungrammaticality of Polarity Items in after-clauses is derived.

Before entering into the details and into the formal definitions, let me first sketch the idea behind the connection between the asymmetry of time branching and the licensing of Polarity Items. One of the most influential approach to the problem of PIs licensing ${ }^{7}$ treats expressions like any as existential quantifiers that lead to a widening of the domain of quantification. In normal, positive contexts, such a widening would cause a loss of information. ${ }^{8}$ In other contexts enlarging the domain brings about a strengthening of the statement made. These kinds of environments share a semantic property - Downward Entailingness - that is, they are characterized by the fact that they enable inferences from set to subsets. The idea is that Polarity Items are legitimate only when they appear in contexts in which the widening of the domain of quantification leads to a strengthening of the claim, i.e., only in downward entailing contexts. Examples of these environments are: negation, antecedents of conditionals, and restrictors of universal quantifiers.

What is then the connection between Polarity Item licensing (i.e., the semantic property of downward entailingness) and the asymmetry of time branching? In the derivation of the necessarily factual interpretation of after-sentences versus the possibly non-veridical instances of before-clauses, we have seen how once we locate a time t (verifying the main

\footnotetext{
${ }^{5}$ That is, for the sentence to be true, at the time in which Fred died it had to be possible that he had a chance to see his grandchildren. The requirement that there is at least one (possible - not necessarily real) future continuation of $\langle\mathrm{w}, \mathrm{t}\rangle$ in which the temporal clause gets realized (that is, the requirement on the definedness of the operator earliest) is meant to rule out anomalous sentences like:

(i) The 7 years-old girl died before she saw her grandchildren.

${ }^{6}$ In a (2004) "aggregate hand-out", Beaver and Condoravdi did present a solution for PI licensing. I analyze their proposal in the Appendix.

${ }^{7}$ See Kadmon and Landman (1993), Krifka (1995), Lahiri (1998) and references therein.

${ }^{8}$ The claim that somelany student came is informationally stronger if the existential quantifier ranges over a "normal" - contextually determined - domain, and it is informationally weaker if the existential quantifier ranges over an enlarged domain of individuals
} 
clause $A$ ), what is past with respect to that $\mathrm{t}$ is instantiated in a single world-history (i.e., only the evaluation world $\mathrm{w}$ is taken into consideration), whereas what is future with respect to $t$ may be realized in different, alternative branches (i.e., a set of historical alternatives is activated). Focussing now on the subordinated clause $B$, it is now straightforward to see that if $B$ is to be located in the past of the $A$-time t (as in the evaluation of $A$ after $B$ ), a single interval of times $\mathrm{t}^{\prime \prime}$ verifying $B$ in $\mathrm{w}$ is to be considered. If on the other hand the clause $B$ is to be (possibly) realized in the future of the $A$-time t (as in the assessment of $A$ before $B$ ), there might be different branches in which $B$ gets instantiated, that is, there might be different intervals of times t" in which $B$ is true. And, in this latter case, there will be many leftboundaries of these $B$-intervals, that is, there will be many earliest times t' that verify $B$. This means that the evaluation of the subordinated clause $B$ requires the assessment of the different forking paths that depart after the $A$-time t. And this is tantamount to saying that it involves an expansion of the domain of possible worlds against which $B$ is evaluated. My claim is that Polarity Items are legitimate in before-clauses precisely because of this enlarging of alternatives. More formally, simply because the $B$-clause now constitutes a downward entailing environments.

Let me now present the formal definition for the uniform analysis of after and before, and then derive the ungrammaticality of Polarity Items in after-clause. With a rough simplification, $A$ before/after $B$ is true iff there is an $A$-time t that precedes/follows all the earliest $B$-times t'. The asymmetric nature of time-branching ensures that in the case of an after-sentence, there is an unique (earliest $B$-time) $t$ '; whereas in the case of a before-clause, there might be different (earliest $B$-time) $\mathrm{t}^{\prime}-$ and for $A$ before $B$ to be true, the $A$-time $t$ must precede all times t'.

More precisely, when $A$ before $B$ is assessed, the event in the $B$-clause follows the event in the $A$-clause, and this amounts to saying that there might be many branches in which $B$ is instantiated (thus, many earliest $B$-times). In order to evaluate $A$ before $B$, we first take into consideration all the time-world pairs $\left\langle\mathrm{w}^{\prime}, \mathrm{t}^{\prime \prime}\right\rangle$ that verify $B$, for any world $\mathrm{w}^{\prime}$ that belongs to the set of historical alternatives to $\mathrm{w}$ at $\mathrm{t}$; and then we collect all the times $\mathrm{t}$ that are the earliest amongst them. The sentence $A$ before $B$ is true in $w$ iff there is an $A$-time $t$ that precedes all the earliest times $t^{\prime}$. In this reformulation of the definition, the temporal clause $B$ constitutes a downward entailing context:

\section{A before B}

$[[A \text { before } B]]^{\mathrm{w}}=1$ iff $\exists \mathrm{t}\left[<\mathrm{w}, \mathrm{t}>\in A \& \forall \mathrm{t}^{\prime}\left[\left(\mathrm{t}^{\prime}=\right.\right.\right.$ earliest. $\left.\left.\left.\lambda \mathrm{t}^{\prime \prime} .\left(\exists \mathrm{w}^{\prime} \in \operatorname{alt}(\mathrm{w}, \mathrm{t})\right)<\mathrm{w}^{\prime}, \mathrm{t}^{\prime \prime}>\in B\right) \rightarrow \mathrm{t}<\mathrm{t}^{\prime}\right]\right]$

(12) We left before anyone came.

[[We left before anyone came] $]^{\mathrm{w}}=1$ iff $\exists \mathrm{t}[\langle\mathrm{w}, \mathrm{t}\rangle \in[[$ we leave $]] \&$

$\forall \mathrm{t}^{\prime}\left[\left(\mathrm{t}^{\prime}=\right.\right.$ earliest. $\lambda \mathrm{t}^{\prime \prime} .\left(\exists \mathrm{w}^{\prime} \in \operatorname{alt}(\mathrm{w}, \mathrm{t})\right)\left\langle\mathrm{w}^{\prime}, \mathrm{t}^{\prime \prime}\right\rangle \in[[$ someone come $\left.\left.\left.]]\right) \rightarrow \mathrm{t}<\mathrm{t}^{\prime}\right]\right]$

$=$ there is a time $t$ such that we leave in $w$ at $t$, and for all times $t^{\prime}$ and for all historical alternatives alt $(w, t) w^{\prime}$ s.t. $t^{\prime}$ is the earliest time in which someone come in $w^{\prime}, t$ precedes $t$ '.

The initial definition for after-sentences mirrors the one for before, with only the direction of temporal ordering reversed.

\section{$A$ after $B$ - def. 1:}

$[[A \text { after } B]]^{\mathrm{w}}=1$ iff $\exists \mathrm{t}\left[\langle\mathrm{w}, \mathrm{t}\rangle \in A \& \forall \mathrm{t}^{\prime}\left[\left(\mathrm{t}^{\prime}=\right.\right.\right.$ earliest. $\left.\left.\left.\lambda \mathrm{t}^{\prime \prime} .\left(\exists \mathrm{w}^{\prime} \in \operatorname{alt}(\mathrm{w}, \mathrm{t})\right)<\mathrm{w}^{\prime}, \mathrm{t}^{\prime \prime}>\in B\right) \rightarrow \mathrm{t}>\mathrm{t}^{\prime}\right]\right]$

But, as Beaver and Condoravdi argued, since the $B$-times t' precede the $A$-time t, the set of historical alternatives is reduced to the evaluation world, thus the definition can be simplified: 
$A$ after $B$ - def. 2:

$[[A \text { after } B]]^{\mathrm{w}}=1$ iff $\exists \mathrm{t}\left[\langle\mathrm{w}, \mathrm{t}\rangle \in A \& \forall \mathrm{t}^{\prime}\left[\left(\mathrm{t}^{\prime}=\right.\right.\right.$ earliest. $\left.\left.\lambda \mathrm{t}^{\prime \prime} .\left\langle\mathrm{w}, \mathrm{t}^{\prime \prime}>\in B\right) \rightarrow \mathrm{t}>\mathrm{t}^{\prime}\right]\right]$

Taking into consideration only a single world, if the after-clause is in fact instantiated in the evaluation world, there is a unique earliest time $t^{\prime}$. Thus, there is no need to universally quantify over all the earliest $B$-times, and thus the definition can be further simplified to:

\section{$A$ after $B$ - def. 3:}

$[[\mathrm{A} \text { after } \mathrm{B}]]^{\mathrm{w}}=1$ iff $\exists \mathrm{t}\left[\langle\mathrm{w}, \mathrm{t}\rangle \in \mathrm{A} \& \mathrm{t}>\right.$ earliest. $\left.\lambda \mathrm{t}^{\prime \prime} .<\mathrm{w}, \mathrm{t}^{\prime \prime}>\in \mathrm{B}\right]$

In this last simplified definition, the after-clause does not constitute anymore a downward entailing context (since the initial universal quantification over earliest $B$-times is reduced to a statement about the unique earliest $B$-time, because of the reduction of alt(w,t) to $\{\mathrm{w}\}$ itself $)$. Thus, Polarity Items are predicted to be ungrammatical in after-clauses.

\section{Conclusion}

With a small amendment to Beaver and Condoravdi's definition for before and after sentences, it is possible to account for the phenomenon of Polarity Items licensing by means of a single lexical schema (i.e., without having to posit two different lexical entries) - that renders only before-clauses a context that licenses strengthening inferences, whereas afterclauses are predicted to create environments in which these inferences do not go through. The difference between before and after is due to the asymmetric nature of time branching - an assumption made by Beaver and Condoravdi to account for their differences in the veridical properties.

\section{Appendix}

\subsection{Linebarger's counterexamples}

Linebarger (1987) noticed how not all instances of Polarity Items in after-clauses lead to ungrammaticality, as witnessed by (13):

(13) He kept writing novels long after he had any reason to believe they would sell.

And the fact that also some after-clauses license Polarity Items constitutes a counterexample to my claim that (after the suitable revisions of the definition) after-clauses are not downward entailing contexts. But before trying to offer a solution, let me cast doubt on the existence of a clearly identifiable class of counterexamples. That is, my question becomes: is there any clear criterion to identify a class of after-clauses that license Polarity Items?

Linebarger herself suggested that these counterexamples had in common the occurrence of an appropriate measure phrase (such as long). But a closer scrutiny demonstrates that the presence of a measure phrase does not constitute neither a necessary (cf. (14)) nor a sufficient condition (cf. (15)) for the licensing of Polarity Items:

(14) Some say the cuts were made after there was any real use for them.

(15) * He kept writing novels long after he retired to any Caribbean island.

Let me moreover notice how the more natural Italian translation of (13) would mark the subordinated clause with subjunctive mood - even if in normal after clauses the indicative is the only viable option:

(16) Ha continuato a scrivere racconti molto dopo che ci fosse alcuna speranza.

(He) has continued to write novels long after that cl. was suBs $_{\text {any hope. }}$ 
And subjunctive mood marking is related to the activation of alternative worlds. Thus, my answer is that, even if I do not have (yet) a clear explanation of the facts, it seems to me that these kinds of sentences require the consideration of alternative branches in which the subordinated clause gets realized - even if the subordinated clause is to be placed in the past of the main clause event.

\subsection{Beaver \& Condoravdi (2004)}

In a (2004) "aggregate" hand out from a series of talks, Beaver and Condoravdi sketch a proposal to explain before and after diverging properties for what concerns Polarity Items licensing. I will first outline Beaver and Condoravdi's argument, ${ }^{9}$ and then I will raise some objections.

Beaver and Condoravdi adopt Kai von Fintel (1999) suggestion, according to which Polarity Items are licensed if strengthening inferences are valid in contexts where all the presuppositions are satisfied. And, since the evaluation of a before-sentence (and an aftersentence) is defined only if the domain of the coercion operator earliest is not empty, we have to check whether strengthening inferences go through in contexts when this presupposition is met, that is, when there is at least a time verifying the subordinated temporal clause.

That is, in order to check whether (18) entails (19), and whether (20) entails (21) - i.e. to check whether before and after create a context in which strengthening inferences are valid we have to consider a context in which (17) is taking for granted (since, if (17) is not assumed, the sentences in (19) and in (21) would turn out as undefined):

(17) At some time, Fred sang loudly.

(18) Everybody left before (=earlier than the first time) Fred sang.

(19) So, everybody left before Fred sang loudly.

(20) Everybody left after (=later than the first time) Fred sang.

(21) $\quad \neq>$ Everybody left after Fred sang loudly.

Beaver and Condoravdi notice how the inferences are secured in the case of a beforesentence, but not when after is involved. This is the case because in the evaluation of $A$ before $B$, the $A$-time t is ordered with respect to the whole event represented by the subordinated clause $B$. And when an event is temporally ordered with respect to a complete interval, then it is temporally ordered with respect to any subpart of it (ad this warrants strengthening inferences). On the other hand, after-clauses are not normally ordered with respect to complete intervals (i.e., an $A$-time may follow the beginning of the $B$-event, without following the whole $B$-event), and this amounts to saying that in that case strengthening inferences are not secured.

Quite interestingly, there are some cases in which the $A$-event is in fact placed after (not just the beginning, but) the whole $B$-event. In those cases, according to Beaver and Condoravdi the coercion operator would pick up the right (and not the left) boundary of the interval corresponding to the $B$-clause (i.e., it would be a latest operator, and not an earliest operator). In these situations, strengthening inferences are indeed valid, and thus Polarity Items are predicted to be grammatical. And these cases would be exemplified by Linebarger's sentences:

(22) He kept writing novels long after he had any reason to believe they would sell.

\footnotetext{
${ }^{9}$ A cautionary remark: I am presenting what I understood of Beaver and Condoravdi's argument - but since my observations are based only on the cited hand out, I might have misunderstood what they meant.
} 
The $A$-event ("he keeps writing novels") is to be placed not simply after the beginning of the $B$-clause, but also after its completion. This licenses strengthening inferences, thus it licenses the occurrence of any.

Summing up, Beaver and Condoravdi propose to connect Polarity Item licensing to contexts that warrants Strawson-like entailments (i.e., strengthening inferences, provided presuppositions are satisfied). And, in normal cases, only before creates such a context, whereas after does not. But there exist also cases in which instead of an earliest operator, a latest operator is at stake: in those cases also after-clauses constitute environments that license strengthening inferences, and thus Polarity Items are predicted to be grammatical, as illustrated by Linebarger's sentences.

I think that Beaver and Condoravdi's analysis is open to some objections. The first one questions their claim that the fact that a time $t$ is temporally ordered with respect to a complete event is a sufficient condition to warrant Strawson-like entailments, and thus to license Polarity Items. Consider for instance an achievement predicate in the $B$-clause:

* He kept writing novels (long) after he retired to any Caribbean island.

An achievement predicate describes a punctual event. In other words, we can say that the earliest time in which "he retired to X" coincide with the whole event of retiring to X. Thus, if the time $t$ in which he keeps writing novels (i.e., in which the main clause is true) follows the earliest time in which he retired to X (i.e., in which the subordinated clause is true), then $t$ will surely follow the whole event of his retiring to $\mathrm{X}$. That is, if there is an achievement predicate in the after-clause, then strengthening inferences ought to go through, and Polarity Items ought to be licensed. But this is not the case, as demonstrated by the ungrammaticality of (23).

Beaver and Condoravdi must have considered such an objection, because in their hand out they take into account (23), and they highlight that: "The act of retirement is punctual. We do not get subset inferences because we are dealing with a singleton set, so the NPI in unlicensed."

But when achievement predicates appear in before-clauses, any is indeed grammatical (that is, NPI are licensed). So, either strengthening inferences ought to go true even if the event denoted by the predicate is punctual, or Polarity Items are not licensed in contexts when subset inferences go through:

(24) Phillip Hazell joined the fray at this time but it took him several seconds before he spotted any German aircraft http://reality.sgiweb.org/suchyta/redbaron/2000/20000211.html

Mr. Brown died, however, before he realized any of his anticipations http://www.rootsweb.com/ nyhchs/townhistories/wilmurt.html

Moreover, there seems to be evidence that the Italian counterpart of after (dopo che) always orders the main clause event with respect to the whole, completed, $B$-event. Thus, for instance, the only reading the Italian (26) receives is that Sandro's arrival in the States follows Gennaro's departure - that is, there cannot be overlapping between the two events:

(26) Sandro è stato in America dopo che Gennaro è stato in America.

Sandro was in America after that Gennaro was in America.

Nevertheless, as witnessed by the example in (9), after-clauses do not license Polarity Items in Italian.

There is another problem connected to Beaver and Condoravdi's explanation for the licensing of Polarity Items in after-clauses. They claim that "in some cases" the coercion operator has to pick up the right boundary (i.e., the latest time) instead of the left boundary (i.e., the earliest time) of an interval of times verifying the subordinated clause. But how are we 
supposed to tell when this is the case? That is, more generally, what are the criteria to set apart cases in which after orders the $A$-event with respect to the earliest $B$-time or with respect to the latest $B$-time? ${ }^{10}$

I have already argued that there are no independent criteria to identify the class of cases in which Polarity Items are legitimate in after-clauses (since the presence of an appropriate measure phrase modifying after (such as long) does not represent neither a necessary nor a sufficient condition - cf. the examples in (14)-(15)). Thus, Beaver and Condoravdi's account turns out to be circular: Polarity Items are licensed in some after-clauses because the $A$-time $\mathrm{t}$ is ordered with respect to the latest $B$-time $t^{\prime}$; but the only reason I could guess for why the $A$ time $\mathrm{t}$ has to be ordered with respect to the latest $B$-time $\mathrm{t}$ ' is simply "because a Polarity Item is grammatical".

More generally, I object to the line of explanation put forth by Beaver and Condoravdi in order to justify before and after diverging licensing properties because I think that it is a more efficient and natural move to resort to the same kind of explanation (i.e., the asymmetric nature of time branching) to account for both veridical and licensing properties. In other words, I hope to have shown that appealing to the same factor (i.e., the asymmetry of time branching) that is held responsible for after's necessarily veridical reading and for before's possibly non-veridical interpretation, it is straightforward to derive as well the licensing of Polarity Items only in before-clauses.

\section{References}

Anscombe, G.E.M.: 1964, Before and after, The Philosophical Review 73, 3-24.

Beaver, D. and Condoravdi, C.: 2003, A Uniform Analysis of Before and After, in R. Young and Y. Zhou (eds.), Proceedings of SALT XIII, CLC Publications, Cornell.

Beaver, D. and Condoravdi, C.: 2004, Before and After in a Nutshell. Aggregate handout from talks presented at Cornell, NYU, MIT and UCSC. http://www.stanford.edu/ dib/

von Fintel, K., 1999: NPI Licensing, Strawson Entailment, and Context Dependency, Journal of Semantics 16, 97-148.

Heinämäki, O.: 1974, Semantics of English Temporal Connectives, Ph.D. thesis, University of Indiana at Bloomington.

Kadmon, N. and Landman, F.: 1993, Any, Linguistics and Philosophy 15, 353-422.

Krifka, M.: 1995, The Semantics and Pragmatics of Polarity Items, Linguistic Analysis 25, 209-257.

Lahiri, U.: 1998, Focus and Negative Polarity in Hindi, Natural Language Semantics 6, 57125.

Landman, F.: 1991, Structures for semantics, Kluwer, Dordrecht.

Linebarger, M.: 1987, Negative Polarity and Grammatical Representation, Linguistics and Philosophy 10, 325-387.

Ogihara, T.: 1995, Non-factual Before and Adverbs of Quantification, in M. Simon and T. Galloway (eds.), Proceedings of SALT V, CLC Publications, Cornell.

\footnotetext{
${ }^{10}$ And why this option is not available in the case of before?
} 